\title{
Luigi Pavia y sus aportaciones a la enseñanza del español para italianos
}

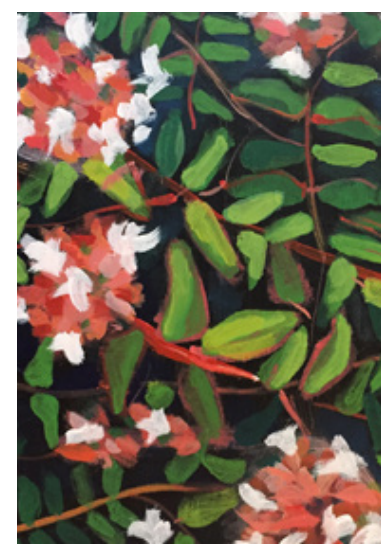

Victoriano Gaviño Rodríguez

Facultad de Filosofía y Letras, Universidad de Cádiz, España /

victoriano.gavino@uca.es

Trabajo recibido el 14 de mayo de 2018 y aprobado el 4 de junio de 2018.

\section{Resumen}

A finales del siglo XIX, Luigi Pavia publicó tres gramáticas españolas (Grammatica elementare della lingua spagnuola con temi, esercizi e letture; Grammatica della lingua spagnuola con temi, letture e dialoghi y Grammatica spagnuola). A pesar de que estas obras fueron editadas posteriormente en varias ocasiones hasta 1927 y se convirtieron en libros de especial importancia en el campo de la enseñanza del español para los italianos, hasta ahora han pasado desapercibidas en el ámbito de la historiografía lingüística. Este trabajo tiene como objetivo mitigar esta falta de atención y analizar la importancia de este autor y su método de enseñanza del español con diferentes objetivos específicos: a) describir sus trabajos, diferentes ediciones, estructura, metodología, objetivos, fuentes; y b) evaluar la importancia de estas gramáticas en el campo de la enseñanza de idiomas, específicamente, la enseñanza del español para italianos entre finales del siglo XIX y principios del siglo XX.

\section{Luigi Pavia and his contributions to the teaching of Spanish to Italians}

\begin{abstract}
At the end of the nineteenth century, Luigi Pavia published three Spanish grammars (Grammatica elementare della lingua spagnuola con temi, esercizi e letture [Elementary grammar of the Spanish language with themes, exercises and readings], Grammatica della lingua spagnuola con temi, letture e dialoghi [Grammar of the Spanish language with themes, readings and dialogues] and Grammatica spagnuola [Spanish grammar]). Even though these works were subsequently published on several occasions until 1927 and became books of special significance in the field of the teaching of Spanish to Italians, so far, they have gone unperceived in the field of linguistic historiography. This work aims to mitigate this lack of attention and
\end{abstract}

Palabras clave

Luigi Pavia gramática española para italianos

historiografía lingüística enseñanza de lenguas enseñanza del español

\section{Keywords}

Luigi Pavia Spanish grammar for Italians linguistic historiography teaching of languages teaching of Spanish 
analyze the significance of this author and his method of teaching Spanish with different specific objectives: a) to describe his works, different editions, structure, methodology, objectives, sources, and b) to evaluate the significance of these grammars in the field of the teaching of languages, specifically, the teaching of Spanish for Italians between the end of the nineteenth century and the beginning of the twentieth century.

\section{Luigi Pavia e suas contribuições para o ensino do espanhol para italianos}

\section{Resumo}

No final do século XIX, Luigi Pavia publicou três gramáticas espanholas (Grammatica elementare della lingua spagnuola con temi, esercizi e letture [Gramática elementar da língua espanhola com temas, exercícios e leituras], Grammatica della lingua spagnuola con temi, letture e dialoghi [Gramática da língua espanhola com temas, leituras e diálogos] e Grammatica spagnuola [Gramática espanhola]). Apesar de que estas obras foram editadas posteriormente em várias ocasiões até 1927 e converteram-se em livros de especial importância no campo do ensino do espanhol para os italianos, até agora passaram despercebidas no âmbito da historiografia linguística. Este trabalho que tem como objetivo mitigar esta falta de atenção e analisar a importância deste autor e seu método de ensino do espanhol com diferentes objetivos específicos: a) descrever seus trabalhos, diferentes edições, estrutura, metodologia, objetivos, fontes e b) avaliar a importância destas gramáticas no campo do ensino de idiomas, especificamente, o ensino do espanhol para italianos entre o final do século XIX e começos do século XX.

\section{Introducción}

Los datos biográficos que hemos podido recopilar de Luigi Pavia son muy escasos. Aunque Lombardini $(2013,37)$ nos informa de que nació en 1854 , el Diccionario bibliográfico fija su fecha de nacimiento dos años después, esto es, en 1856: "Pavia Luigi, eminente letterato e professore, nato a Milano nel 1856. Studioso, colto, scrittore forbito, profondo in varie lingue, oltre a gran numero di articoli su giornali e riviste, ha fatto numerose pubblicazioni molto lodate dalla stampa e da critici illustri" (Rovito 1907, 192). Ejerció de profesor en varios lugares, a juzgar por las informaciones que aportan las portadas de algunas de sus gramáticas: "professore nel Ro. Istituto Tecnico di Brescia" (Pavia 1894, 1895a y 1895b), "professore nei Ro. Istituto Tecnico di Mantova" (Pavia, ${ }^{2} 1901$ ) y "professore nei Ri. Insituti Tecnici d'Italia" (Pavia ${ }^{3} 1907,{ }^{4} 1913,{ }^{5} 1921,{ }^{6} 1927$ ). A estos puestos, hay que añadir que también enseñó durante bastante tiempo en el Instituto técnico de Nápoles (Rovito 1907, 192). Su producción escrita fue considerable, incluyendo, entre otros, novelas, poesías e importantes trabajos de lingüística ${ }^{1}$, así como varias gramáticas del inglés, alemán, italiano y español ${ }^{2}$.

En lo referente a la gramática española, Pavia es autor de tres tratados, titulados Grammatica elementare della lingua spagnuola con temi, esercizi e letture (publicado por primera vez en 1894), Grammatica della lingua spagnuola con temi, letture e dialoghi (1895a) y Grammatica spagnuola

\section{Palavras-chave}

Luigi Pavia gramática espanhola para italianos

historiografia linguística ensino de línguas ensino do espanhol

Algunos de estos títulos son los siguientes: Pan per focaccia, L'ultima Lotta, Le nozze d' Irma, Ferma in posta, Valentina Visconti, II Romanzo del sentimento, Hissn al-Gebel, Grido d'Upupa, Fra Note di Musica Turca, Guida Illustrativa di Salsomaggiore, Storia municipale di Crema, Storia municipale di Lodi, Storia municipale di Brescia, Il Fatto di Rezzato, Monumenti della poesia Casligliana del Medio Evo, La lingua Castigliana ne' suoi primordî, Appunti sulla Romanza primitiva nella Spagna, Il Cid e i suoi tempi, Considerazioni sulle Scuole Italiane in Oriente, Interessi Italiani in Levante, II Dizionario dell'Academia Jugoslava, Croazia ed Ungheria nella lotta fra Diritto e Despotismo, Introduzione allo studio della lingua inglese.

2. Como indica Lombardini (2013, 37-38), Luigi Pavia fue autor de muchos textos dedicados a la didáctica de distintas lenguas y otros temas afines: publicó para el inglés Corso di lingua inglese ad uso degli italiani: introduzione: squardo generale alla grammatica ed esercizi relativi (1881), Grammatica inglese (1884), Chiave dei temi contenuti nella grammatica inglese (1891), Nuova grammatica elementare della lingua inglese: con temi, letture, e dialoghi (1888), Grammatica della, lingua inglese: con temi letture e dialoghi (189o[?]) y su versión española Gramática de la lengua inglesa: con ejercicios de versión, lecturas y diálogos (21908); para el alemán, Esegesi della grammatica tedesca (1891), Grammatica tedesca (1893), Esercizi di traduzione, con vocabolario a complemento della grammatica tedesca (1894), Esercizi tedeschi, con richiami grammaticali e note elucidative (1900), Corso moderno di Lingua tedesca, ad uso degli italiani (1910); para el italiano, Gramática sucinta de la lengua italiana (1908). 
(1895b), todos ellos concebidos de manera conjunta y complementaria para el aprendizaje de nuestra lengua desde distintos niveles de complejidad y profundización. Ninguna de estas obras ha recibido hasta el momento la atención debida por parte de los especialistas, de modo que este estudio intentará llevar a cabo un análisis de los aspectos más sobresalientes de la serie textual formada por este grupo de gramáticas españolas. Los objetivos concretos del trabajo se centran en lo siguiente: a) presentar la estructura global e ideas gramaticales de cada una de estas obras (y sus diferentes ediciones); b) examinar las fuentes en las que se basa Pavia para el desarrollo de su doctrina gramatical; y c) evaluar el alcance de estos trabajos, así como la relevancia de Luigi Pavia en el terreno de la didáctica de la enseñanza del español para italianos.

\section{Las gramáticas españolas de Pavia}

Solo por el número de ediciones de sus gramáticas españolas a lo largo de más de treinta años, puede afirmarse que estamos ante una de las figuras más destacadas en el ámbito de la enseñanza del español para italianos en el siglo XIX. En total, hay constancia de que fueron publicadas trece ediciones de sus tres gramáticas españolas entre 1894 y 1927, fechas de la primera edición de la Grammatica elementare della lingua spagnuola y de la última edición de la Grammatica della lingua spagnuola, respectivamente. El alto promedio de aparición de estas obras es en sí mismo un dato suficientemente revelador y que nos da una clara idea de la importancia que tuvo la figura de Pavia a partir de finales del siglo XIX, coincidiendo con la culminación del proceso de unificación de Italia y el auge de los flujos migratorios hacia Hispanoamérica ${ }^{3}$. No obstante, y a diferencia de otros textos de la época, en estos no aparece mención o referencia alguna a los destinatarios ni en el título ni en los prefacios de estas obras, por lo que, aun estando ante gramáticas orientadas específicamente a la enseñanza del español para italianos, sus destinatarios podían ser variados: emigrantes que salían de Italia, personas que necesitaban la lengua española como medio de comunicación (especialmente, viajeros o comerciantes que precisan de esta lengua para sus negocios o necesidades comunicativas cotidianas), aquellos que simplemente optaban por aprender español como un aspecto más de su formación culta como individuo, etcétera.

Los tres tratados gramaticales que estudiamos son monolingües escritos en italiano, lo que en buena medida demuestra - como sostiene A. Sánchezque "el verdadero auge de las gramáticas de español para extranjeros surge en el momento en que sus autores deciden escribir esas gramáticas en la lengua de aquellos a quienes iban destinadas" (Sánchez 1992, 41). Al tratarse de obras escritas expresamente para hablantes de italiano como lengua materna, tienen en cuenta los aspectos más particulares o conflictivos del aprendizaje del español en contraste con el italiano. Tal y como comenta el propio autor en dos de estas gramáticas, sus obras iban destinadas a todos aquellos interesados en aprender español después de un periodo de prejuicios sobre ella:

Deposti omai, da molti, taluni imperdonabili pregiundizî che rispetto alla bellissima lingua spagnuola erano, fino a pochi anni fa, troppo comuni fra gl'Italiani, questi si sono messi ora volonterosi ed in buon numero allo studio di quella lingua, che, sorella germana della loro, tanto le assomiglia, e le sta a paro sotto ogni rispetto (Pizarro 1895a y 1901, III)4.
3. La importancia de su obra podría situarse al mismo nivel de la de $\mathrm{M}$. Pizarro y la publicación de su Metodo teorico-pratico per imparare la lingua spagnuola ad uso degl'italiani, que fue editado por vez primera en $1873 \mathrm{y}$ publicado con diferentes nombres (y en ocasiones de manera anónima) al menos en $1877,{ }^{3} 1881,{ }_{1}^{4} 1884,{ }^{5} 1886,{ }^{6} 1894$, ${ }^{6} 1896,{ }^{10} 1900-1901$ y ${ }^{11} 1914$. En todo el siglo XIX no tenemos otro precedente igual salvo el de la figura de Francesco Marin, que, tras la primera y única edición de 1833 en Roma de L'italiano istruito nella cognizione della lingua spagnuola, publicó otras ocho ediciones milanesas de esta obra bajo el título de Grammatica della lingua spagnuola o sia l'italiano istruito nella cognizione di questa lingua (concretamente en 1837 . $1853,1860,1862,1867,1869,1874$ y 1885 ).
4. A partir de 1907 , con leves variaciones en la redacción, aunque conservándose plenamente el mismo mensaje: "Deposti omai, da molti, certi imperdonabili pregiundizî che, rispetto alla bellissima lingua spagnuola, fino a pochi anni fa, erano troppo comuni fra gl'Italiani, questi si sono messi ora volonterosi ed in buon numero allo studio di quella lingua, che, sorella germana della loro, tanto le assomiglia, e le sta a paro sotto ogni rispetto" (Pavia ${ }^{1} 1907$ b, III; 51921, III y ${ }^{6} 1927$, III). 
Como en otros casos, estamos ante textos que salen a luz por el impulso y la iniciativa editorial de ciertos impresores, en este caso, Giulio Groos y Ulrico Hoepli, que intentaban completar sus colecciones de gramáticas de lenguas extranjeras con estas dedicadas a la lengua española ${ }^{5}$.

\subsection{Ediciones, modelos y estructura global de las obras}

\subsubsection{Grammatica elementare della lingua spagnuola con temi, esercizi e letture}

La Grammatica elementare della lingua spagnuola con temi, esercizi e letture es la primera gramática española publicada por Pavia en 1894. Esta obra tuvo diferentes reediciones: una segunda en 1901 y una tercera edición en 1907, todas ellas publicadas en Heidelberg por G. Groos ${ }^{6}$. Como su propio nombre indica, esta gramática elemental aborda de manera sucinta los entresijos gramaticales de nuestra lengua y va destinada a aquellos que quieran acercarse a ella de manera inicial. Su autor así lo manifiesta, al señalar lo siguiente: "benchè elementare, potrà bastar tuttavia a soddisfare le esigenze di coloro che vogliono occuparsi della bellissima lingua di Spagna senza addentrarsi nelle minute particolarità grammaticali della stessa" (Pavia 1894, III). Para aquellos que quieran profundizar más en su estudio, Pavia aconseja en su prefacio el uso de su otra gramática, más extensa y completa, esto es, la Grammatica della lingua spagnuola con temi, letture e dialoghi (1895a), que se publicaría en el año siguiente, pero en la que (a juzgar por sus palabras), estaría trabajando este autor o incluso tendría ya finalizada a la par que esta ${ }^{7}$. Quizás por su carácter de obra elemental (con poco más de 200 páginas), la obra se articula desde su primera edición de manera muy sencilla en tres partes: en las dos primeras se presenta específicamente la doctrina gramatical (la primera parte está destinada a los aspectos gráficos y fonéticos; la segunda, al estudio de la morfología — como la denomina el propio Pavia—, distribuida en 36 lecciones dedicadas al estudio de las distintas clases de palabras). La gramática se completa con una tercera parte (no numerada como tal) en la que encontramos diversos aspectos didácticos (ejercicios y temas promiscuos [sic] consistentes en diversos textos de traducción al español y al italiano, frases fáciles, trozos de lectura, un apéndice con información sobre la moneda española y, finalmente, un vocabulario italiano-español y otro español-italiano) que ocupan en total más de 50 páginas. No existe en la obra un capítulo dedicado a la sintaxis. En sus distintas ediciones, la estructura de la gramática obedece a un modelo primario, el de la edición prínceps de 1894, repetido en la segunda edición de ${ }^{2} 1901$ a y levemente alterado en el submodelo de ${ }^{3} 1907$, como puede observarse en el siguiente esquema:

\begin{tabular}{|l|l|}
\hline Modelo 1894 y $^{2} 1901 a$ (205 + VIII pp.) & Submodelo ${ }^{31907 a ~(210 ~+~ V I I ~ p p .) ~}$ \\
\hline Prefazione (pp. III-IV) & Dalla prefazione alla 1 1 $^{\underline{a}}$ edizione (pp. III-IV) \\
Indice (pp. V-VII) & Alla nuova edizione (p. IV) \\
Errata-Corrige (p. VIII) & Indice (pp. V-VII) \\
Parte I. Grafia e Fonetica (pp. 1-12) & Errata-Corrige (p. VII) \\
Parte II. Morfologia (pp. 13-149) & Parte I. Grafia e Fonetica (pp. 1-12) \\
Ejercicios y temas promiscuos (pp. 150-154) & Parte II. Morfologia (pp. 13-173) \\
Frases fáciles (pp. 155-157) & \\
Trozos de lectura (pp. 157-171) & Appendice. Monete Spagnuole (p. 173) \\
Appendice. Monete Spagnuole (p. 171) & Vocabolario (pp. 174-210) \\
Vocabolario (pp. 172-205) & \\
\hline
\end{tabular}

Tabla 1: Ediciones, modelos, estructuras y páginas de la Grammatica elementare della lingua spagnuola con temi, esercizi e letture.
5. Así se infiere además de las palabras del propio Pavia (1894, III): “Fra le numerose grammatiche pubblicate dalla solerte casa editrice Julius Groos di Heidelberg non poteva nè doveva omai più mancarne una della lingua spagnuola per gl'Italiani”.

6. Frente a la tendencia habitual, la Grammatica elementare y la Grammatica della lingua spagnuola son publicadas en el extranjero. No sucede lo mismo con el tercero de estos textos, la Grammatica spagnuola, que sale a luz en Milán.

7. En cualquiera de los casos, es interesante reseñar aquí que las elaboraciones y redacciones de estas tres gramáticas no son independientes; estamos ante un único proyecto homogéneo concebido por su autor de manera conjunta y complementaria para el estudio de nuestra lengua. 
A pesar de que en su tercera edición Pavia afirma haber llevado a cabo cambios y mejoras en todo el libro ${ }^{8}$, lo cierto es que las modificaciones que se dan a nivel teórico son menores, afectando, fundamentalmente, a la parte práctica, en la que se realiza un cambio en la disposición de las lecturas ${ }^{9}$, se introducen nuevos ejercicios o se amplía el vocabulario.

\subsubsection{Grammatica della lingua spagnuola con temi, letture e dialoghi}

Un año más tarde, según reza en portada (aunque con escasa diferencia de meses de la anterior) sale a la luz la Grammatica della lingua spagnuola con temi, letture e dialoghi (1895), la obra de mayor envergadura y alcance de las tres gramáticas que aquí tratamos, a juzgar no solo por las 6 ediciones que llegó a publicar el editor Giulio Groos en Heidelberg de la misma en los años 1895a, ${ }^{2} 1901$ b, ${ }^{3} 1907 b,{ }^{4} 1913,{ }^{5} 1921$ y ${ }^{6} 1927$ (con una vigencia de 32 años entre la primera y última de sus ediciones) ${ }^{10}$, sino también por su carácter de obra más completa y cerrada desde el punto de vista organizativo y doctrinal, pues reúne, con mayor nivel de detalle, la descripción gramatical del español en un libro que dobla el número de páginas al anterior. La estructura de la obra obedece al siguiente esquema:
8. L. Pavia ( $31907 a$, IV) nos dice lo siguiente: "Questa nuova edizione della mia Grammatica Elementare della Lingua Spagnuola esce ritoccata e migliorata in tutto il corso del libro, tanto per l'esposizione teoretica [...] quanto per i temi di versione che riformai in molta parte".

9. En las dos primeras ediciones de la obra, el apartado de frases fáciles y lecturas aparecen de manera independiente, pero a partir de la tercera edición estos contenidos son incluidos en el interior de las lecciones, junto a otros ejercicios.

\begin{tabular}{|c|c|c|}
\hline $\begin{array}{l}\text { Modelo } 1895 a y^{2} 1901 b \\
(422+\text { VIII pp. })\end{array}$ & $\begin{array}{l}\text { 1er submodelo }{ }^{3} 1907 \mathrm{~b} \\
(428+\text { VIII pp.) }\end{array}$ & $\begin{array}{l}2^{0} \text { submodelo }{ }^{41} 1913 ;{ }^{51} 1921,{ }^{6} 1927 \\
(414+\text { VIII pp. })\end{array}$ \\
\hline $\begin{array}{l}\text { Prefazione (pp. III-IV) } \\
\text { Indice (pp. V-VIII) } \\
\text { Parte I. Grafia e Fonetica (pp. 1-22) } \\
\text { Parte II. Morfologia (pp. 23-218) } \\
\text { Parte III. Usi delle parole e sintassi } \\
\text { (pp. 219-344) } \\
\text { Parte IV. Esercizî varî pp. 345-422) }\end{array}$ & $\begin{array}{l}\text { Dalla prefazione alla } 1^{\underline{a}} \text { edizione } \\
\text { (pp. III-IV) } \\
\text { Alla nuova edizione (pp. IV) } \\
\text { Indice (pp. V-VIII) } \\
\text { Parte I. Grafia e Fonetica (pp. 1-22) } \\
\text { Parte II. Morfologia (pp. 23-222) } \\
\text { Parte III. Usi delle parole e sintassi } \\
\text { (pp. 223-346) } \\
\text { Parte IV. Esercizî varî (pp. 347-427) } \\
\text { Errata-Corrige (p. 428) }\end{array}$ & $\begin{array}{l}\text { Dalla prefazione alla } 1^{\underline{a}} \text { edizione (pp. III-IV) } \\
\text { Alla nuova edizione (pp. IV) } \\
\text { Indice (pp. V-VIII) } \\
\text { Parte I. Grafia e Fonetica (pp. 1-24) } \\
\text { Parte II. Morfologia (pp. 25-224) } \\
\text { Parte III. Usi delle parole e sintassi } \\
\text { (pp. 225-344) } \\
\text { Parte IV. Esercizî varî (pp. 345-414) }\end{array}$ \\
\hline
\end{tabular}

Tabla 2: Ediciones, modelos, estructuras y páginas de la Grammatica della lingua spagnuola con temi, letture e dialoghi.

Las estructuras de las distintas ediciones de esta gramática se ciñen todas al modelo de la edición prínceps de 1895a, que recibe modificaciones en los submodelos iniciados por la tercera y la cuarta edición, aun cuando estos presentan escasas variaciones, como puede deducirse de la propia estructura y paginación de la obra que reza en la tabla anterior. En este sentido, en el primer submodelo, el propio Pavia nos anuncia ( $\left.{ }^{3} 1907 \mathrm{~b}, \mathrm{IV}\right)$ la presencia de estos cambios, centrados, específicamente, en el aparato práctico, donde se eliminan y añaden algunos poemas con respecto al modelo inicial, hay cambios en las traducciones y diálogos, aparece el vocabulario italiano-español y español-italiano con nuevas entradas, etcétera. En el submodelo iniciado por Pavia $\left({ }^{5} 1921\right)$ también se insiste en cambios en esta misma línea, ya que se incorporan algunas indicaciones varias sobre aspectos comunicativos de especial importancia (como los saludos, las horas, los paseos, los viajes, el tiempo, etcétera) y se eliminan los textos de lectura de prosa, por deseo del propio editor, tal y como confiesa el propio Pavia (51921, IV) en su prefacio.
10. En relación con la existencia de una segunda edición milanesa editada por Hoepli también en 1901 de la que da noticias Lombardini $(2013,38$ n. 63) conviene apostillar que esta edición concreta no parece que exista como tal; los ejemplares que podrían corresponderse con esta publicación son exactamente los de la edición alemana, en cuya portada Ulrico Hoepli sobrepuso con un adhesivo sus datos editoriales en el pie de imprenta para la difusión de este tratado en Italia. Por otro lado, y con respecto a una supuesta tercera edición de esta obra en 1913, ubicada en B. del Centro interdip. di servizi di Palazzo Maldura dell'U. degli studi di Padova de Padua y B. del Centro italiano documentazione azione studi de Turín (Lombardini 2013, 576), parece ser que estamos nuevamente ante un error, al menos a juzgar por la información que aportan los catálogos de tales bibliotecas, que solo contemplan una cuarta edición de la obra en 1913. Sí existe, sin embargo, una tercera edición de la obra en 1907 . no mencionada en Lombardini (2013). 
La segunda edición de la obra es una reimpresión exacta de la edición prínceps de 1895a, y lo mismo puede decirse de las ediciones de ${ }^{5} 1921,{ }^{6} 1927$, que reproducen el texto de la cuarta edición de ${ }^{4} 1913$.

En esta ocasión, y aunque la obra no opta por la división tradicional de la gramática en cuatro partes (Prosodia, Ortografía, Analogía y Sintaxis) seguida por muchas gramáticas españolas de la época ${ }^{11}$, sí aparece en el texto una estructura más acorde a estos cánones, con tres partes, dedicadas a grafía y fonética, morfología y sintaxis, respectivamente ${ }^{12}$. No falta, por último, en la obra el aspecto didáctico, tan prolífico en la época en este tipo de trabajos y que queda de manifiesto en las gramáticas de Pavia no solo en la presencia constante de ejercicios, sino también en la ausencia de discusiones teóricas, todo ello en favor de una descripción gramatical directa, normalmente no muy detallada de la lengua, en la que se suelen resaltar los elementos divergentes entre el español y el italiano, usando para ello ejemplos y textos con una marcada intención didáctica. En definitiva, y aunque de manera diversa, el autor intenta dar su mejor respuesta a la demanda de los aprendientes de la lengua en la época, seleccionando para ello las preguntas que considera pertinentes para la gente en su momento, en su contexto histórico ${ }^{13}$.

\subsubsection{Grammatica spagnuola}

La Grammatica spagnuola, por último, es la tercera de las gramáticas que analizamos en este estudio, si bien su primera edición aparece en el mismo año que la anterior, esto es, 1895, y tan solo unos meses después de la primera edición de la Grammatica elementare de $1894^{14}$. Esta última gramática tuvo cuatro ediciones: $1895 \mathrm{~b},{ }^{2} 1904,{ }^{3} 1912$ y ${ }^{4} 1919$. A diferencia de las anteriores, la Grammatica spagnuola se publicó en Milán, de la mano del editor Hoepli. Todas las ediciones respetan la filosofía y estructura de la edición prínceps, por lo que podemos afirmar que existe un único modelo gramatical de la obra en 1895, siendo el resto de ediciones submodelos de este con pequeños cambios que afectan a la paginación o suponen leves modificaciones en el contenido. La estructura, contenidos y paginación de cada edición quedan recogidos en la siguiente tabla:

\begin{tabular}{|l|l|}
\hline Modelo 1895b (194 + XI pp.) & 1er submodelo ${ }^{21904}(194+$ XI pp.) \\
\hline Prefazione (pp. VII-VIII) & Prefazione alla 1. - Edizione (p. V) \\
Indice (pp. IX-XI) & Prefazione alla 2.․ Edizione (p. VII) \\
Capo I. Fonetica e grafia (pp. 1-14) & Indice (pp. IX-XI) \\
Capo II. Particolarità fonico-grafiche (pp. 15-19) & Capo I. Fonetica e grafia (pp. 1-14) \\
Capo III. Articolo e sostantivo (pp. 20-42) & Capo II. Particolarità fonico-grafiche (pp. 15-19) \\
Capo IV. L'aggettivo qualificativo (pp. 43-63) & Capo III. Articolo e sostantivo (pp. 20-42) \\
Capo V. I Pronomi (pp. 64-86) & Capo IV. L'aggettivo qualificativo (pp. 43-63) \\
Capo VI. Numeri (pp. 87-100) & Capo V. I Pronomi (pp. 64-86) \\
Capo VII. II verbo (pp. 101-178) & Capo VI. Numeri (pp. 87-100) \\
Capo VIII. Parole inflettibili (pp. 179-185) & Capo VII. II verbo (pp. 101-178) \\
Letture (pp. 186-194) & Capo VIII. Parole inflettibili (pp. 179-185) \\
Alcune interjezioni (p. 194) & Alcune interjezioni (p. 185) \\
& Letture (pp. 186-194) \\
\hline
\end{tabular}

11. Para una revisión de esta y otras divisiones en las gramáticas españolas a lo largo del periodo estudiado, cf. los estudios de J. J. Gómez Asencio (1981) y M. ${ }^{a}$ L. Calero Vaquera (1986). Esta última investigadora señala en el periodo comprendido entre 1847 y 1920 hasta un total de veintiocho seguidores de esta división (Calero Vaquera 1986, 40).

12. En este caso, no se prescinde de la sintaxis y se afronta su estudio, aun cuando se realiza desde una perspectiva tradicional, centrada en el estudio de la palabra y su combinación con otras.

13. Precisamente para satisfacer la necesidad de aquellos que estudiaban español de manera autónoma, la Grammatica della lingua spagnuola con temi, letture e dialoghi se publicaba acompañada de un volumen separado con las claves para los ejercicios, titulado Chiave dei Temi.

14. El texto definitivo de esta última gramática está fechado en octubre de 1894 , tal y como reza en el prefacio de su primera edición (cf. Pavia 1895, VII). 


\begin{tabular}{|c|c|}
\hline $2^{2}{ }^{\circ}$ submodelo ${ }^{31912}(194+\mathrm{XI}$ pp.) & 3er submodelo ${ }^{4} 1919(231+$ XII pp.) \\
\hline $\begin{array}{l}\text { Prefazione alla } 1^{\text {a }} \text { Edizione (p. V) } \\
\text { Prefazione alla } 3^{\text {a }} \text { Edizione (p. VII) } \\
\text { Indice (pp. IX-XI) } \\
\text { Errata-Corrige (p. XII) } \\
\text { Capo I. Fonetica e grafia (pp. 1-14) } \\
\text { Capo II. Particolarità fonico-grafiche (pp. 15-19) } \\
\text { Capo III. Articolo e sostantivo (pp. 20-42) } \\
\text { Capo IV. L'aggettivo qualificativo (pp. 43-63) } \\
\text { Capo V. I Pronomi (pp. 64-86) } \\
\text { Capo VI. Numeri (pp. 87-100) } \\
\text { Capo VII. II verbo (pp. 101-178) } \\
\text { Capo VIII. Parole non flessive (pp. 179-185) } \\
\text { Alcune interjezioni (p. 185) } \\
\text { Letture (pp. 186-194) }\end{array}$ & $\begin{array}{l}\text { Prefazione alla Prima Edizione (p. V) } \\
\text { Prefazione alla Terza Edizione (p. VI) } \\
\text { Prefazione alla Quarta Edizione (p. VII) } \\
\text { Indice (IX-XI) } \\
\text { Errata-Corrige (p. XII) } \\
\text { Capo I. Fonetica e grafia (pp. 1-19) } \\
\text { Capo II. Particolarità fonico-grafiche (pp. 20-26) } \\
\text { Capo III. Articolo e sostantivo (pp. 27-52) } \\
\text { Capo IV. L'aggettivo qualificativo (pp. 53-75) } \\
\text { Capo V. I Pronomi (pp. 76-103) } \\
\text { Capo Vl. Numeri (pp. 104-120) } \\
\text { Capo VII. II verbo (pp. 121-206) } \\
\text { Capo VIII. Parole non flessive (pp. 207-219) } \\
\text { Alcune interjezioni (p. 220) } \\
\text { Letture (pp. 221-231) }\end{array}$ \\
\hline
\end{tabular}

Tabla 3: Ediciones, modelos, estructuras y páginas de la Grammatica spagnuola.

Algunas observaciones al respecto de estas obras:

a) En lo referente a los paratextos, todas las ediciones cuentan con un índice de contenidos al comienzo, así como los correspondientes prefacios, breves, redactados por el propio Pavia, en los que el autor presenta las novedades de cada edición ${ }^{15}$. Frente a las otras gramáticas de este autor, estamos ante una "grammatichetta" que, dada su extensión, es muy directa en sus planteamientos y se centra en los aspectos más importantes y esenciales de la lengua, sin adentrarse en temas teóricos, y cuya estructura organizativa prescinde de divisiones en partes. En su primera edición, Pavia (1895b, VII) nos presenta su texto: "Presento una grammatica molto elementare della lingua spagnuola, nella quale tuttavia ho cercato di concentrare ed esporre chiaramente ilustrandole con molti esempi, le regole necessarie per imparare la morfologia, la fonetica e la grafia"16. Los prefacios de la segunda y tercera edición indican que el texto apenas ha recibido innovaciones, salvo las correspondientes a las correcciones de las escasas inexactitudes presentes en la anterior edición ${ }^{17}$. Por último, la cuarta edición (Pavia ${ }^{4} 1919$ ) supone una ampliación de la obra, como puede verse en el aumento del volumen y paginación de la obra, además de las palabras del propio Pavia $\left({ }^{4} 1919, \mathrm{VII}\right)$, que en el prefacio a esta cuarta edición afirma lo siguiente: "Infatti ho aggiunta una quantitá di nuove spegazioni teoretiche, di nuovi esempî ed esercizî, affinchè il libro riesca non più tanto elementare come per lo innanzi".

b) En todas sus ediciones, la Grammatica spagnuola articula su cuerpo central en ocho capítulos: los dos primeros tratan aspectos fonéticos y gráficos; los cinco siguientes abordan cada uno de ellos un tema de morfología, en concreto, una clase de palabras.

c) En la medida en que se lo permite su pequeño formato y extensión, esta gramática aporta los habituales contenidos didácticos de los textos de esta época, que en esta ocasión consiste en abundantes ejemplos, ejercicios para los diferentes temas abordados ${ }^{18}$, así como un capítulo específico con lecturas en español y otro (muy breve) en el que se presentan algunas interjecciones de nuestra lengua con su correspondiente traducción al italiano.
15. Como elemento peculiar, puede señalarse que todos los prefacios siguen presentes en las ediciones sucesivas, a excepción del de la segunda, que es eliminado a partir de la tercera edición. Así, 1895b conserva el prefacio a la primera edición; 21904 el de la primera y segunda; ${ }^{3} 1912$, el de la primera y tercera, $y^{4} 1919$, el de la primera, tercera y cuarta edición.

16. A continuación, el autor anima a aquellos que quieran adentrarse mejor en el estudio de la lengua española a que consulten sus otras dos gramáticas, publicadas por Groos en Heidelberg.

17. Los distintos submodelos de 1904 1912 guardan gran similitud con el texto original pero no pueden considerarse reediciones de este, pues albergan algunas modificaciones, eso sí, de escasa importancia, como señala el propio autor en los prefacios específicos a ambas ediciones (cf. Pavia ${ }^{2} 1904$ VII y ${ }^{31912,}$ VII): "Nulla di essenziale è stato innovato nella presente edizione; tuttavia ritoccai, si può dire, tutto il libro, levandone le poche inesattezze ch'erano sfuggite nella $2^{\mathrm{a}}$ edizione".

18. En esta ocasión, no tenemos un vocabulario, si bien los distintos ejercicios suelen incluir listas de vocabulario en dos columnas. 


\subsection{Doctrina gramatical: Fuentes e influencias}

Las tres obras examinadas guardan indudables puntos de contacto entre sí, no solo por el escaso espacio temporal en el que se gestan los textos, sino también por su consideración como obras complementarias de un proyecto único, que sirven para el aprendizaje del español en distintos niveles. Desde el punto de vista doctrinal, las ideas gramaticales de Pavia en estas obras entroncan con la tradición grecolatina, teniendo, eso sí, como referencias los textos de autores de la tradición gramatical italiana, en especial, las gramáticas del español y/o del italiano como lenguas extranjeras de Miranda (1566), Franciosini (1624) o Veneroni (1681), que gozaron de gran éxito y fueron imitadas por las gramáticas italianas publicadas a partir del XVII.

Aunque estamos ante tratados gramaticales, en las tres obras predomina una clara vertiente didáctica, que se visualiza por la presencia constante de elementos didácticos (lecturas de textos, diálogos, vocabularios...) tan frecuentes en la época en el terreno de la enseñanza de lenguas ${ }^{19}$. Con esta práctica, estos tratados intentan reflejar "con naturalidad el lenguaje usado en la calle o el propio de algunas profesiones y ocupaciones habituales en la sociedad de entonces: comprar, vender, en el mercado, buscar posada, preguntar por el camino para viajar de un lugar a otro, etc.” (A. Sánchez 1992, 16).

Estamos en una época en la que las imprentas suelen marcar sus tratados de enseñanza de idiomas con algún método del que, generalmente, posee los derechos de uso, de ahí que, en muchos casos, estas obras de corte didáctico, aunque no encuentran marco teórico homogéneo, son vinculadas unívocamente a alguno de estos métodos, que son publicitados en sus portadas (o en el propio título) como garantes de calidad de las obras en un periodo en el que el carácter comercial y los beneficios económicos para la imprenta con este tipo de trabajos comienzan a considerarse un factor determinante. En el caso concreto de Pavia, dos de sus gramáticas (la Grammatica elementare della lingua spagnuola con temi, esercizi e letture y la Grammatica della lingua spagnuola con temi, letture e dialoghi) aparecen relacionadas con el método Gaspey-Otto-Sauer para el estudio de las lenguas modernas ${ }^{20}$, cuyas bondades son puestas de manifiesto por el propio editor en algún lugar visible del libro, por ejemplo, su contraportada, y en la que se relaciona la calidad de su método con las numerosas y frecuentes ediciones de gramáticas redactadas según un método de "fama mundial" para la enseñanza de lenguas (Pavia 1895a).

El propio Pavia (1895a, III) afirma que, por deseo del editor, se ha basado en la Spanish Conversation Grammar de C. M. Sauer para los ejercicios, las lecturas o la propia disposición de la materia, un libro destinado a aquellas personas que quieren aprender la lengua desde un punto de vista práctico y en el que el autor presenta las reglas de manera sencilla y fácil ${ }^{21}$. Y de hecho, la estructura del índice de la Grammatica della lingua spagnuola con temi, letture e dialoghi y algunos de sus contenidos son bastante fieles al libro de Sauer, de ahí que la obra de Pavia sea también deudora de algunas influencias indirectas recibidas por medio de Sauer, cuya doctrina gramatical está basada —así lo dice su autor (Sauer ${ }^{71904, ~ I I I-I V) — ~ e n ~ l a s ~ g r a m a ́ t i c a s ~ d e ~ G i l d o, ~ G i r o ́ ~}$ y Roma, Bello y Salvá. Estamos ante un gramático por encargo, que no tiene pudor alguno en reconocer la imposición de sus fuentes, así como disculparse por los posibles defectos o errores de su obra. En este sentido, el propio Pavia termina su prólogo de manera modesta manifestando la esperanza de que su trabajo pueda responder a sus propósitos didácticos.
19. Todas las obras se centran, específicamente, en aspectos descriptivos, sin detenerse en aspectos teóricos. Como el propio Pavia (1895a, IV) sostiene en uno de sus prefacios, intenta presentar la materia de manera clara y simple: "ho cercato di esporre la materia in modo semplice, chiaro ed opportuno, non mancando di fare i debiti raffronti ove fosse conveniente, ma astenendomi d'altro lato da tutte quelle superfluità che sono rese innecessarie dalla grande simiglianza formale e sintattica delle due lingue, spagnuola ed italiana".

20. El método Gaspey-Otto-Sauer aparece como exclusivo del editor Giulio Groos, de Heidelberg, y así reza en una advertencia aparecida en la contraportada de estas dos gramáticas de Pavia (cf., por ejemplo, sus dos primeras ediciones de 1894 y 1895 a).

21. En el caso de la Grammatica spagnuola, Pavia nos menciona también a Sauer y su obra Spanish Conversation Grammar: "Il presente libro nella redazione del quale, aderendo ad un desiderio dell'editore, ove fu opportuno e conveniente mi servii, sia per esercizî, specie di lettura, sia per la disposizione della materia, della Spanish Conversation Grammar di C. M. Sauer consta di quattro parti" (Pavia 1895, III). Como fuente de la Gramática elemental, en especial para la parte de ejercicios prácticos y la lista de vocabularios, Pavia (1894, III) admite haber seguido la Spanish Grammar for Germans, publicada por C. M. Sauer y H. Runge. 
Partiendo de los ejemplares que he podido consultar de estas obras, algunos de estos textos (al menos, con seguridad, las ediciones de ${ }^{2} 1901 \mathrm{~b},{ }^{3} 1907 \mathrm{~b}$ y ${ }^{4} 1913$ de la Grammatica della lingua spagnuola con temi, letture e dialoghi y la edición de 1907a de la Grammatica elementare della lingua spagnuola con temi, esercizi e letture) vienen con dos mapas ilustrativos: el primero es un mapa de España que se sitúa al comienzo del libro; el otro, un mapamundi en el que se especifican las zonas donde se habla español. En esta línea, estas obras siguen la línea editorial de Groos, que también en la Spanish Conversation Grammar de C. M. Sauer (71904) incluye mapas similares ${ }^{22}$.

Frente a los anteriores, la Grammatica spagnuola salió a la luz en Milán —ya lo he advertido previamente- y su método se enmarca dentro de la línea de los manuales que Ulrico Hoepli publicó para la enseñanza de lenguas extranjeras. Estamos ante lo que hoy en día podría denominarse un libro de bolsillo, donde la adscripción metodológica no es especialmente importante: no hay alusión a ninguna fuente, aunque se alude a las dos obras anteriores del propio autor (Pavia 1895b, VII).

No pretendo examinar en detalle la doctrina de Pavia en estas obras, pero sí conviene hacer algunas reflexiones generales sobre algunos de los aspectos más importantes de su teoría gramatical en diversos apartados de sus gramáticas.

En el ámbito específico de la fonética y la grafía, todas las obras intentan dar cuenta, en pocas líneas, de las dificultades fundamentales que para un italiano presenta el aprendizaje de la lengua española. No destaca Pavia por ser un autor original entre los de tratados de enseñanza del español para italianos, si bien su planteamiento es singular en algunos puntos. Así, por ejemplo, junto a otros autores de la época, Pavia considera la existencia de 28 letras en el alfabeto, si bien es el único del siglo XIX que no menciona la $k$ pero sí la $r r$ en este sistema ${ }^{23}$. Su propuesta global, que incluye $a, b$, $c, c h, d, e, f, g, h, i, j, l, l l, m, n, \tilde{n}, o, p, q, r, r r, s, t, u, v, x, y, z$ (entre otros, Pavia, 1894, 1; 1895a, 1 y 1895b, 1), difiere también de la de Sauer, al que Pavia declara (aunque quizás por imposición editorial) fuente de su obra ${ }^{24}$.

Pasamos al ámbito específico de la morfología. Una vez que la gramática académica abandona en 1870 el sistema de nueve clases de palabras por uno de diez ${ }^{25}$, son muchas las gramáticas españolas que siguen esta clasificación ${ }^{26}$. Entre estos, L. Pavia (1894, 13 y 1895a, 25), junto a otros como L. Zuccaro (1881) y G. Schilling y F. Demattio (1890), propone a finales del XIX dividir las clases de palabras en diez, si bien su propuesta es única, porque elimina de la lista general el participio (lo incluye en las formas verbales) y considera como una clase individual los números: artículo, sustantivo, adjetivo calificativo, pronombre, número, verbo, adverbio, preposición, conjunción, interjección ${ }^{27}$. En Pavia $(1895 b, 14)$ se varía levemente la denominación para dos de estas categorías: el sustantivo y el adjetivo calificativo pasan a denominarse nombre sustantivo y nombre adjetivo, respectivamente, siguiendo una tradición terminológica que también tiene su arraigo en algunas obras de nuestra tradición gramatical. Como ya advertí previamente, las gramáticas de Pavia carecen de definiciones y caracterizaciones teóricas, de modo que las presentaciones de cada una de estas partes del discurso (según la terminología utilizada por Pavia) se suelen limitar a la presentación de la formación de estos elementos en español, así como a las distintas clasificaciones internas, problemas de flexión y traducción existentes para cada una de ellas.
22. En el caso de Sauer, no contamos con un mapamundi al final de la obra, sino un mapa de América del Sur y América central, lo que podría darnos pistas acerca de los destinatarios principales de este texto. 23. Otros tratados de enseñanza de español para italianos del XIX que presentan también un sistema de 28 letras son los de Blanc (1847), Catá (1870), Monaci y D'Ovidio (1879), Anónimo (1884), Manetta y Rughi (21891), Gorra (1898), Anónimo (1898) y Foulques (1898). Frente a estos, la obra temprana de Borroni (1812) nos habla de 22 letras ( $a, b, c, d, e, f, g, h, i$ I, $m, n, o, p, q, r, s, t,[u v], x, y, z) ;$ Marin (1833 y 1837) y Pizarro $\left(1873,1877,{ }^{3} 1881\right.$, ${ }^{6} 1894$ y $\left.{ }^{6} 1896\right)$ contemplan la existencia de 26 ( $a, b, c, d, e, f, g, h, i, j, l, I l, m, n$, $\tilde{n}, o, p, q, r, s, t, u, v, x, y, z)$, mientras que una concepción más cercana a la de Pavia es la representada por el sistema de 27 letras defendido por Marin $\left({ }^{2} 1853,{ }^{3} 1860,{ }^{4} 1862,{ }^{7} 1874 y^{8} 1885\right)$, Anónimo (1869), Richeri (1871-1872), Manetta y Rughi (1872-1873), Gaffino (1879), Zuccaro (1881) y Schilling y Demattio (189o): $a, b, c, c h, d, e, f, g, h$, $i, j, l, l l, m, n, \tilde{n}, o, p, q, r, s, t, u, v, x, y, z$.

24. En este punto, Sauer $\left({ }^{1} 1904,1-2\right)$ se muestra seguidor de la propuesta académica que, a partir de RAE (1870, 1-2), defiende la existencia de las siguientes 28 letras: $a, b, c, c h, d, e, f, g$, $h, i, j, k, I, I l, m, n, \tilde{n}, o, p, q, r, s, t, u, v, x$, $y, z$. Conviene puntualizar, no obstante que el problema de la consideración de $k$ como elemento del alfabeto no ha estado claro en el ámbito de la Academia, ya que su presencia o no en el alfabeto español ha fluctuado a lo largo de las distintas publicaciones académicas... (continúa en página 31)

25. Para la RAE (1796, 3-4), “TODAS las palabras de que nos valemos para declarar nuestros pensamientos son, y se llaman partes de la oracion: las quales son nueve por este órden: artículo, nombre, pronombre, verbo, participio, adverbio, preposicion, conjuncion, interjección". En RAE $(1870,5)$ se afirma que "se reducen todas, para facilitar el análisis, á diez clases, Ilamadas comunmente partes de la oracion, y son á saber: artículo, nombre, adjetivo, pronombre, verbo, participio, adverbio, preposicion, conjuncion é interjeccion".

26. El sistema de nueve clases, que había sido defendido en Italia por importantes autores como Miranda (1566, 12), Franciosini $(1624,5)$ y Veneroni $(1681$, s.p.), ejerce gran influencia en algunas obras del XIX (y más adelante), que siguen contemplando la existencia de nueve partes de la oración: $B$. Borroni (1812), S-H. Blanc (1847), M. Pizarro $\left(1873,1877,{ }^{3} 1881,{ }^{6} 1894 y^{6}{ }^{6} 1896\right)$ y Anónimo (1884 y 1898) hablan de artículo, nombre, pronombre, verbo, participio, adverbio, preposición, conjunción e interjección. Más minoritaria aunque también con seguidores, es la defensa de ocho partes (nombre, pronombre, verbo, participio, adverbio, preposición, conjunción, interjección) en F. Marin $\left(1833 ; 1837\right.$; ${ }^{2} 1853$; ${ }^{31860}$; $\left.41862 y^{7} 1874 y^{8} 1885\right)$ y G. Catá (1870). 27. L. Zuccaro (1881), G. Schilling y F. Demattio (1890) son más tradicionales y categorizan las clases en artículo, nombre, adjetivo, pronombre, verbo, participio, adverbio, preposición, conjunción e interjección. 
La doctrina sintáctica es la que menos tratamiento recibe en las obras de Pavia. De hecho, este apartado solo está presente en la Grammatica della lingua spagnuola con temi, letture e dialoghi. En cualquier caso, este aspecto (que podríamos ver como defecto de su obra) es una constante en la época. En la mayor parte de gramáticas de este momento, la sintaxis constituye un mero añadido a la parte de Analogía con el que se intenta tratar con mayor profundidad la combinatoria de las distintas partes de la oración, sin especiales novedades ni aportaciones. Se trata, por lo general, de una parte menor y anecdótica, a la que estos tratados prestan escasa atención. La obra de Pavia no es una excepción en este punto.

\section{Concluyendo}

Luigi Pavia es un claro exponente de la historia de la enseñanza del español para italianos. Las numerosas ediciones publicadas de su obra atestiguan que su método de enseñanza del español tuvo una amplia difusión en el periodo entre finales del siglo XIX y principios del XX, momento crucial en el que comienza a proliferar el interés por el estudio de lenguas extranjeras y del español en particular para los italianos. Su teoría gramatical, aunque vertebrada de manera práctica en su obra, es heredera en gran parte de la tradición gramatical italiana anterior; presenta escasas novedades doctrinales, pues apoya fundamentalmente su trabajo en los aspectos didácticos, que, como se ha visto por las modificaciones que reciben las distintas ediciones gramaticales, siguen gobernando las tendencias de enseñanza y aprendizaje de la lengua en este periodo.

\section{Notas}

24 En este punto, Sauer $(71904,1-2)$ se muestra seguidor de la propuesta académica que, a partir de RAE (1870, 1-2), defiende la existencia de las siguientes 28 letras: $a, b, c, c h, d, e$, $f, g, h, i, j, k, l, I I, m, n, \tilde{n}, o, p, q, r, s, t, u, v, x, y, z$. Conviene puntualizar, no obstante, que el problema de la consideración de $k$ como elemento del alfabeto no ha estado claro en el ámbito de la Academia, ya que su presencia o no en el alfabeto español ha fluctuado a lo largo de las distintas publicaciones académicas: en el Diccionario de Autoridades (RAE 1734, 339, 1) se dice que es la "UNDECIMA letra en el orden de nuestro Alphabéto" aunque se advierte que podría excluirse de nuestro alfabeto porque su pronunciación se podría suplir con c o q. Esta postura perdura en el $\operatorname{DRAE}(1780,569,1)$ y años más tarde, en 1803 es considerada ya la duodécima letra (tras la incorporación de ch). Sin embargo, a partir del $\operatorname{DRAE}(1817,512,1-2)$ se dice de manera confusa lo siguiente de $k$ : "Esta letra, que se ha contado hasta ahora como perteneciente a nuestro abecedario, solo se usa en algunas voces tomadas de otros idiomas, y aun en estas se puede suplir con la $C$ antes de las vocales $\mathrm{A}, \mathrm{O}, \mathrm{U}$ y con la $\mathrm{Q}$, interponiéndose la $\mathrm{U}$ antes de la $\mathrm{E}, \mathrm{l}$ ". Y esta es la visión que aparece hasta el $\operatorname{DRAE}(1869,451,1)$, en el que vuelve a considerarse como duodécima letra del alfabeto castellano y novena de las consonantes. Para un estudio acerca de la influencia ejercida por la Real Academia Española en la doctrina gramatical aparecida en los tratados de enseñanza del español para italianos en el siglo XIX, véase Gaviño Rodríguez (2014). (En página 30.) 


\section{Q Bibliografía}

"Anónimo. 1869. Nuovo metodo teorico-pratico per imparare la lingua spagnuola secondo il metodo di F. Ahn. Milano: Paolo Carrara.

"Anónimo. 1884. Il nuovo Franciosini. Grammatica della lingua spagnuola ad uso degl' italiani. Milano: Oreste Ferrario.

"Anónimo. 1898. Il nuovo Franciosini. Grammatica della lingua spagnuola ad uso degli italiani. Milano: Casa Editrice Guigoni.

"Blanc Saint-Hilaire, Marie Jean. 1847. Nuovo franciosini o sia Grammatica della lingua spagnuola all'uso degli italiani. Parigi e Lione: Libreria Cormon e Blanc.

"Borroni, Bartolomeo. 1812. Nuovissima grammatica spagnuola compilata da Bartolomeo Borroni ad uso degl'italiani. Milano: Giovanni Silvestri.

»Calero Vaquera, María Luisa. 1986. Historia de la gramática española (1847-1920): De A. Bello a R. Lenz. Madrid: Gredos.

"Catá, Giu. 1870. Nuovissimo Franciosini ossia Grammatica della lingua spagnuola per uso degli italiani. adatta al metodo del dottore Ollendorff. Barcelona, Buenos Aires: Carlo Alou.

"Foulques, Alessandro Edoardo. 1898. Grammatica della lingua spagnola. Milano, BuenosAyres: Casa Editrice Angelo Bietti.

》Franciosini, Lorenzo. 1624. Grammatica spagnola, e italiana, hora nuovamente uscita in luce, Mediante la quale può il Castigliano con facilità, e fondamento impadronirsi della lingua Toscana, \& il Toscano, della Castigliana. Venetia: Giacomo Sarzina.

》Gaffino, Francesco. 21879. Metodo di Ollendorff per imparare a leggere, scrivere e parlare una lingua. Ammaestramento per imparare la lingua spagnuola all'uso degli italiani. Francoforte: Carlo Jügel, Librajo-Editore.

" Gaviño Rodríguez, Victoriano. 2014. "Influencias de la Real Academia Española en tratados de enseñanza del español para italianos en el siglo XIX". En Perfiles para la historia crítica de la gramática del español en Italia: siglos XIX y XX, editado por Félix San Vicente, Lourdes de Hériz, Ana y María Enriqueta Pérez Vázquez, 223-239, Bologna: Bononia University Press.

"Gómez Asencio, José Jesús. 1981. Gramática y categorías verbales en la tradición española (1771-1847). Salamanca: Ediciones Universidad de Salamanca.

"Gorra, Egidio. 1898. Lingua e letteratura spagnuola delle origini. Milano: Hoepli.

» Lombardini, Hugo Edgardo. 2013. “Gramáticas para la enseñanza del español en la Italia del siglo XIX: el caso de Francesco Marin”. Tesis doctoral, Universidad de Salamanca.

»Manetta, Filippo y Edoardo Rughi. 1872-1873. Grammatica della Lingua spagnuola. Roma, Torino, Firenza: Ermanno Loescher.

》Manetta, Filippo y Edoardo Rughi. 21891. Grammatica della lingua spagnuola. Torino: Ermanno Loescher.

》 Marin, Francesco. 1833. L'italiano istruito nella cognizione della lingua spagnuola. Roma: Salviucci.

"Marin, Francesco. 1837. Gramatica della lingua spagnuola o sia l'italiano istruito nella cognizione di questa lingua. Milano: Giovanni Silvestri. 
" Marin, Francesco. ${ }^{2} 1853$. Gramatica della lingua spagnuola o sia l'italiano istruito nella cognizione di questa lingua. Seconda edizione milanese notabilmente corretta e ampliata dall'Ab. Pietro Monti. Milano: Tipografia e Libreria di Giovanni Silvestri.

》Marin, Francesco. ${ }^{31860}$. Grammatica della lingua spagnuola o sia l'italiano istruito nella cognizione di questa lingua. Milano: Silvestri.

》Marin, Francesco. ${ }^{41862}$. Grammatica della lingua spagnuola ossia l'italiano istruito nella cognizione di questa lingua. Quarta edizione milanese diligentemente riveduta aggiuntavi una serie di temi composti dal D’Luigi Monteggia. Milano: Antica Casa Libraria Giov. Silvestri ora di L. Recchioni.

"Marin, Francesco. ${ }^{1} 867$. Grammatica della lingua spagnuola, ossia l'italiano istruito nella cognizione di questa lingua. Quinta edizione milanese diligentemente riv. aggiuntavi una serie di temi composti di Luigi Monteggia. Milano: Libreria di Educazione e d'Istruzione di Paolo Carrara.

" Marin, Francesco. ${ }^{6} 1869$. Grammatica della lingua spagnuola, ossia l'italiano istruito nella cognizione di questa lingua. Sesta edizione milanese diligentemente riv. aggiuntavi una serie di temi composti di Luigi Monteggia. Milano: Libreria di Educazione e d'Istruzione di Paolo Carrara.

"Marin, Francesco. ${ }^{7874 .}$. Grammatica della lingua spagnuola ossia l'italiano istruito nella cognizione di questa lingua. Settima edizione milanese riveduta e corretta secondo la ultima decisioni dell'Accademia Spagnuola dal professore Giacomo Richeri. Milano: Libreria di Educazione e d'Istruzione di Paolo Carrara.

" Marin, Francesco. ${ }^{8} 1885$. Grammatica della lingua spagnuola o sia l'italiano istruito nella cognizione di questa lingua. Milano: Carrara.

»Miranda, Giovanni. 1566. Osservationi della lingua castigliana, divise in quatro libri: ne quali s'insegna con gran facilita la perfetta lingua spagnuola. Con due tavole: l'una de capi essentiali, $\varepsilon$ l'altra delle cose notabili. Vinegia appresso Gabriel Giolito de'Ferrari.

"Monaci, Ernesto y Francesco D'Ovidio. 1879. Manualetti d'introduzione agli studj neolatini, composti per uso degli studenti delle Facoltà di Lettere. I. Spagnolo. Napoli: Edizione a spese degli autori.

》Pavia, Luigi. 1894. Grammatica elementare della lingua spagnuola con temi, esercizî e letture. Heidelberg: Giulio Groos.

»Pavia, Luigi. 1895a. Grammatica della lingua spagnuola con temi, letture e dialoghi. HeideIberg: Giulio Groos.

» Pavia, Luigi. 1895b. Grammatica spagnuola. Milano: Ulrico Hoepli.

"Pavia, Luigi. 21901a. Grammatica elementare della lingua spagnuola con temi, esercizî e letture. Heidelberg: Giulio Groos.

"Pavia, Luigi. ${ }^{21901 b . ~ G r a m m a t i c a ~ d e l l a ~ l i n g u a ~ s p a g n u o l a ~ c o n ~ t e m i, ~ l e t t u r e ~ e ~ d i a l o g h i, ~ S e c o n-~}$ da edizione. Heidelberg: Giulio Groos.

"Pavia, Luigi. ${ }^{21904 . ~ G r a m m a t i c a ~ s p a g n u o l a . ~ S e c o n d a ~ e d i z i o n e ~ r i v e d u t a ~ d a l l ' A u t o r e . ~ M i-~}$ lano: Ulrico Hoepli.

"Pavia, Luigi. ${ }^{31907 a . ~ G r a m m a t i c a ~ e l e m e n t a r e ~ d e l l a ~ l i n g u a ~ s p a g n u o l a ~ c o n ~ t e m i, ~ e s e r c i z i ̂ ~ e ~}$ letture. Terza edizione rilavorata e aumentata dall'autore. Heidelberg: Giulio Groos.

» Pavia, Luigi. ${ }^{31907 b}$. Grammatica della lingua spagnuola con temi, letture e dialoghi. Terza edizione. Heidelberg: Giulio Groos.

" Pavia, Luigi. 31912. Grammatica spagnuola. Terza edizione riveduta dall'Autore. Milano: Ulrico Hoepli. 
"Pavia, Luigi. 41913. Grammatica della lingua spagnuola con temi, letture e dialoghi. Quarta edizione. Heidelberg: Giulio Groos.

"Pavia, Luigi. 41919. Grammatica spagnuola. Quarta edizione riveduta e ampliata dall'Autore. Milano: Ulrico Hoepli.

» Pavia, Luigi. 51921. Grammatica della lingua spagnuola con temi, letture e dialoghi. Quinta edizione. Heidelberg: Giulio Groos.

» Pavia, Luigi. ${ }^{6}$ 1927. Grammatica della lingua spagnuola con temi, letture e dialoghi. Sesta edizione. Heidelberg: Giulio Groos.

"Pizarro, Mattia. 1873. Metodo teorico-pratico per imparare la lingua spagnuola ad uso degl'italiani secondo i metodi moderni. Milano: Giovanni Gnocchi, Editore.

"Pizarro, Mattia. 1877. Nuovo metodo facile teorico-pratico per imparare la lingua spagnuola secondo il metodo di F. Ahn. Milano: Giovanni Gnocchi, Editore.

»Pizarro, Mattia. 31881. Nuovo metodo teorico-pratico per imparare la lingua spagnuola secondo il metodo di F. Ahn. Terza edizione corretta e migliorata con aggiunte. Milano: Giovanni Gnocchi, Editore.

"Pizarro, Mattia. ${ }^{41884}$. Nuovo metodo teorico-pratico per imparare la lingua spagnuola secondo il metodo di F. Ahn. Quarta edizione corretta e migliorata con aggiunte. Milano: Giovanni Gnocchi, Editore.

"Pizarro, Mattia. 51886. Nuovo metodo teorico-pratico per imparare la lingua spagnuola secondo il metodo di M. Pizarro. Quinta edizione correta e migliorata con aggiunte. Milano: Giovanni Gnocchi.

"Pizarro, Mattia. ${ }^{6} 1894$. Nuovo metodo teorico-pratico per imparare la lingua spagnola secondo il metodo di F. Ahn. Sesta edizione corretta e migliorata con aggiunte. Milano: Giovanni Gnocchi Editore.

》 Pizarro, Mattia. 61896. Nuovo metodo teorico-pratico per imparare la lingua spagnuola secondo il metodo di F. Ahn. Sesta edizione corretta e migliorata con aggiunte. Milano: Giovanni Gnocchi, Editore.

» Pizarro, Mattia. ${ }^{10} 1900-1901$. Nuovo metodo teorico-pratico per imparare la lingua spagnuola secondo il metodo di F. Ahn. Milano: Giovanni Gnocchi.

»Pizarro, Mattia. ${ }^{11} 1914$. Nuovo metodo teorico-pratico per imparare la lingua spagnuola secondo il metodo di F. Ahn. Milano: Giovanni Gnocchi.

" Rovito, Teodoro. 1907. Dizionario bio-bibliografico dei letterati e giornalisti italiani contemporanei. Napoli: Tipografia Melfi \& Joele.

"Real Academia Española. 1734. Diccionario de la lengua castellana, en que se explica el verdadero sentido de las voces, su naturaleza y calidad, con las phrases o modos de hablar, los proverbios o refranes, y otras cosas convenientes al uso de la lengua. Dedicado al Rey Nuestro Señor Don Phelipe V (que Dios guarde), à cuyas reales expensas se hace esta obra. Compuesto por la Real Academia Española. Tomo quarto. Que contiene las letras G.H.I.J.K.L.M.N. Con privilegio. Madrid: Imprenta de la Real Académia Españóla.

"Real Academia Española. 1780. Diccionario de la Lengua castellana compuesto por la Real Academia Española, reducido á un tomo para su mas fácil uso. Madrid: D. Joachin Ibarra, Impresor de Cámara de S.M. y de la Real Academia.

»Real Academia Española. 1796. Gramática de la lengua castellana. Quarta edicion corregida y aumentada. Madrid: Viuda de D. Joaquin de Ibarra.

"Real Academia Española. 1817. Diccionario de la Lengua castellana por la Real Academia Española. Quinta edición. Madrid: Imprenta Real. 
》Real Academia Española. 1869. Diccionario de la Lengua castellana por la Academia Española. Undécima edición. Madrid: Imprenta de don Miguel Rivadeneyra.

"Real Academia Española. 1870. Gramática de la lengua castellana. Nueva edición, corregida y aumenta. Madrid: Imprenta y estereotipia de M. Rivadeneyra.

"Richeri, Giacomo. 1871-1872. Nuovo metodo teorico-pratico per imparare la lingua spagnuola secondo il metodo di F. Ahn. Milano: Libreria di Educazione e di Istruzione di Paolo Carrara.

》Sánchez Pérez, Aquilino. 1992. Historia de la enseñanza del español como lengua extranjera. Madrid: Sociedad General Española de Librería, S. A.

"Sauer, Charles Marquard. 71904. Spanish Conversation-Grammar. Heidelberg, Boston: Julius Groos-Schoenhof Book Company.

»Schilling, Giulio y Fortunato Demattio. 1890. Grammatica spagnola con ispeciale riguardo alla lingua parlata. Lipsia, Turin: G. A. Gloeckner editore, H. Loescher's Hofbuchhandlung.

"Veneroni, Giovanni. 1681. Le maître italien: Contenant tout ce qui est nécessaire pour apprendre facilement, $\&$ en peu de temps, la Langue Italienne. Paris.

»Zuccaro, Luigi. 1881. Grammatichetta spagnuola. Milano: Societá Editrice Sonzogno. 